O OPEN ACCESS
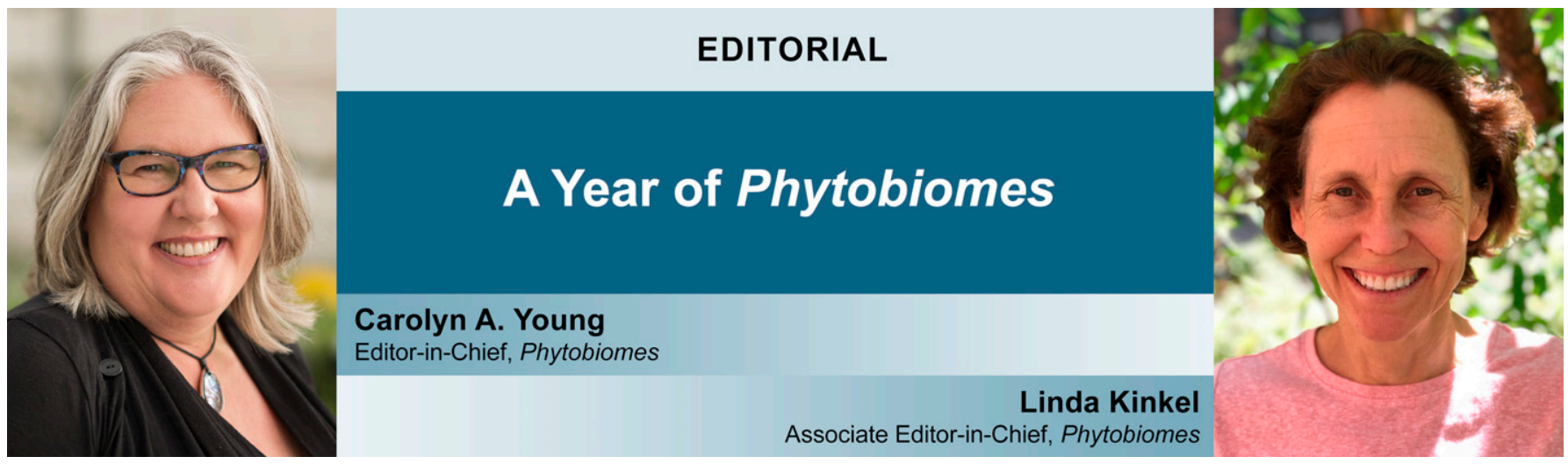

Carolyn A. Young, Noble Research Institute, LLC, Ardmore, OK 73401; and Linda L. Kinkel, Department of Plant Pathology, University of Minnesota, St. Paul, MN 55108

Accepted for publication 16 May 2018.

It is hard to believe that we have just celebrated the one-year anniversary of our first issue of the Phytobiomes journal! As we celebrate our first year, and as the term 'phytobiome' becomes more deeply embedded in our science, it is worth pausing to reflect on the impetus and science behind the phytobiome concept, and on the next steps for our journal.

\section{THE PHYTOBIOME CONCEPT}

In 2015, The American Phytopathological Society (APS) launched the Phytobiome Initiative, which posed a challenge to our community to expand our thinking to pursue integrated, multiscale, ecological systems approaches that would enhance our understanding and management of plant biomes. The phytobiome concept provokes us to push the boundaries of our science to think bigger. While single factor experiments that isolate the effects of individual variables on disease have served and continue to serve us well, the phytobiome concept seeks to understand and then harness the diversity and complexity of biotic and abiotic interactions that result in a productive plant. The capacity to move beyond the study of individual variables or interactions is explicitly enabled by recent scientific advances. In particular, the vast increase in data generation capacity facilitated by diverse 'omics technologies, particularly in characterizing plant microbiomes; enhanced low-cost and high-resolution environmental monitoring capacities; dramatic increases in the availability and sophistication of systems biology and multiscale modeling platforms; and the potential for precision agriculture-enabled technologies to support translation of these

(C) 2018 The American Phytopathological Society titanic data streams to practical crop management are all critical to the development of phytobiome science. We have much work to do to accomplish the vision established by the Phytobiomes Roadmap (www.phytobiomes.org/roadmap). However, the development of creative and innovative phytobiome science promises to stimulate new approaches for study, provide novel insights into plants across their biomes, and promote the development of inventive management approaches that capitalize upon understanding phytobiome complexity rather than simplification, as a keystone of success.

In charting the path forward, it is worth re-emphasizing the definition of the phytobiome. While often used interchangeably with the plant microbiome, the phytobiome concept is much bigger than 'just' plants and their microbes. As noted in the Phytobiomes Roadmap and by others, "The phytobiome is composed of plants, their environment, and diverse interacting microscopic and macroscopic organisms, which together influence plant health and productivity" (Leach et al. 2017; www.phytobiomes.org/roadmap). With a strong commitment to the phytobiomes concept, APS moved forward to sponsor a new journal, Phytobiomes, to create a publication outlet and foundation for research in this area. The explicit definition of phytobiomes matters, as it defines the scope of phytobiome research and (not coincidentally) the scope of publications in the Phytobiomes journal. While disease is an important part of the phytobiome, phytobiome studies are inherently complex, and seek to simultaneously evaluate multiple biotic and abiotic components of plant systems. This inclusion of multiple factors is one feature that distinguishes publishing in the Phytobiomes journal from publication in other APS journals.

We note the challenges to authors and reviewers in expanding the complexity of our experimental designs, research systems and questions. Many of us have not been trained to tackle the simultaneous study of plants, microbes, insects, soil, weather, and their 
interactions. Sampling multiple components of the phytobiome, and at spatial and temporal scales that capture the dynamics of individual components and their relationships to plant productivity, can be extraordinarily difficult. Additionally, rapid developments in 'omics technologies and analytical pipelines have led to correspondingly large shifts in expectations for microbiome data size and analyses, making it a challenge for authors and reviewers to keep up. The Phytobiomes journal, through our authors, reviewers, and editorial board, is committed to engaging actively with the community as our collective expectations and standards for phytobiome science evolve. There will be growing pains, but our goal and commitment is nothing less than leadership in creating and maintaining the highest standards in building the science needed to harness complex data to optimize phytobiome productivity.

\section{A NEW PUBLISHING FRAMEWORK: RESOURCE ANNOUNCEMENTS}

Considering the landscape of data generation and publishing, APS editors recently moved forward to develop a new manuscript type, Resource Announcement, which is available in all APS journals. Resource Announcements offer our community the opportunity to publish a brief overview of significant resources that have been generated and are now available, including for example genome sequences and plant or crop microbiomes. The first of these can be found in Molecular Plant-Microbe Interactions, with an announcement presenting the genome sequences of Phytophthora pathogens of strawberry and raspberry (Tabima et al. 2017). Phytobiomes recently published its first Resource Announcements, one highlighting the availability of microbiome data from citrus infected with 'Candidatus Liberibacter asiaticus' (Ginnan et al. 2018), and the second, a genome from the plant growth-promoting fungus Serendipita vermifera subsp. bescii (Ray et al. 2018). Additional types of Resource Announcements are anticipated across APS journals as the complexity and long-term value of specific resources continues to grow.

Resource Announcements provide two different opportunities. First, they allow easier citation of a data set. For example, many microbiome or genome studies are likely to result in multiple publications, often from collaborating research groups. Publishing your data in a Resource Announcement will make this accessible without delay and frees up space in the big paper of results you are planning. Secondly, some of our early microbiome studies don't have enough samples or the sampling strategy is not conducive for the conclusions that are stated. By placing your data in a Resource Announcement, you are still able to make your data available to the wider scientific community. To find out more, check out the authors instructions (https://apsjournals.apsnet.org/page/authorinformation).

Finally, as we begin our second year, this is a good opportunity to thank a lot of people who have helped make Phytobiomes a success. Our editorial team, the Senior Editors who handle your manucripts, and the Associate Editors, are a diverse group of scientists that help you get your work published. They provide direction for the publication to get through what sometimes feels like an onerous review process, but they have got your back and want to see your manuscript succeed. The reviewers who have answered the call and provided guidance to improving manuscripts have also been critical to our success. The Publications team who had to work out all the kinks of starting a new journal, from the submission pages to the design and layout of each article and much more, have been invaluable. We thank all the authors who have taken a chance with a new journal and wanted to publish with us. Your papers are what makes Phytobiomes a success. And finally, thanks to you, the readers! I hope you have enjoyed the first year. Thank you for checking us out, downloading articles, and citing our work. We look forward to our next year, and to reading your best work in Phytobiomes.

\section{LITERATURE CITED}

Ginnan, N. A., Dang, T., Bodaghi, S., Ruegger, P., Peacock, B. B., McCollum, G., England, G., Vidalakis, G., Roper, C., Rolshausen, P. E., and Borneman, J. 2018. Bacterial and fungal next generation sequencing datasets and metadata from citrus infected with 'Candidatus Liberibacter asiaticus'. Phytobiomes 2:64-70.

Leach, J. E., Triplett, L. R., Argueso, C. T., and Trivedi, P. 2017. Communication in the phytobiome. Cell 169:587-596.

Ray, P., Chi, M. H., Guo, Y., Chen, C., Adam, C., Kuo, A., LaButti, K., Lipzen, A., Barry, K. W., Grigoriev, I. V., Tang, Y., and Craven, K. D. 2018. Genome sequence of the plant growth-promoting fungus Serendipita vermifera subsp. bescii: The first native strain from North America. Phytobiomes 2:62-63.

Tabima, J. F., Kronmiller, B. A., Press, C. M., Tyler, B. M., Zasada, I. A., and Grünwald, N. J. 2017. Whole genome sequences of the raspberry and strawberry pathogens Phytophthora rubi and P. fragariae. Mol. PlantMicrobe Interact. 30:767-769. 